\title{
What Can Pre-Main Sequence Binary Star Populations Tell Us about Binary Formation Mechanisms?
}

\author{
Andrea M. Ghez \\ University of California Los Angeles \\ Department of Physics and Astronomy \\ Los Angeles, CA 90095-1562
}

\begin{abstract}
We review current observations of binary star populations with particular attention to what insight these populations can give us into the problem of how binary stars form. Significant progress has been made in the past few years, revealing variations as a function of site, primary star mass, and binary star separations. The variations in the binary star population with type of star formation site in comparison with the field, suggests that $\sim 30 \%$ of the field binaries formed in loose $\mathrm{T}$ associations and $\sim 70 \%$ formed in the dense progenitors of open clusters. Variations with mass and separation on the whole are well matched by the predictions of fragmentation followed by competitive accretion. However, there remains much work to be done on both the observational and theoretical end before a complete picture of binary star formation can be developed.
\end{abstract}

\section{Introduction}

The wealth of young low mass binary stars identified over the last decade with the advent of high spatial resolution imaging techniques has presented a number of challenges to star formation theory. First, since binary stars appear to be the norm as opposed to the exception, it is clear that the primary products of star formation are multiple stars and not single stars. This demands that the simplifying assumption of spherical symmetry be abandoned in models of the star formation process. Second, the majority of the observed binary star systems have separations that are smaller than the size typically assumed for a circumstellar disk ( 100 AU) and are, on average, comparable to the distance between the Sun and Neptune $(\sim 30 \mathrm{AU})$. Interactions between companion stars and the circumstellar disk environment must therefore be considered in models of planetary formation. Third, in nearby $\mathrm{T}$ associations the companion star fraction appears to be a factor of $\sim 2$ greater than that of the solar-type main sequence stars found in the solar neighborhood (Ghez et al. 1993, 1997; Leinert et al. 1993; Simon et al. 1995; Köhler \& Leinert 1998). This unexpected result raises a number of questions about possible variations between different sites of star formation as well as the relationship of these $\mathrm{T}$ Association regions to the final field population. This review focuses on the current progress in our observational picture of binary star formation. 


\section{Companion Star Fractions}

A number of questions are raised by the factor of two discrepancy in the binary star frequencies between the pre-main sequence stars in the nearby star forming regions and the main sequence stars in the solar neighborhood at separation of tens to hundreds of AU. Is the difference real or simply an artifact of varying sensitivities to low mass companions? This effect might arise due to the shape of the evolutionary tracks, which map a given mass ratio into a smaller luminosity ratio at an age of $2 \mathrm{Myr}$ compared to $4.5 \mathrm{Gyr}$ (e.g., Burrows et al. 1998). Therefore, it is possible that $\mathrm{T}$ Tauri star surveys are detecting brown dwarf companions that are relatively more luminous when they are young. Initially, it was quite difficult to address this question, since obtaining secondary masses from single wavelength measurements can lead to erroneous mass estimates. Several multi-wavelength studies are now being carried out allowing for robust mass estimates (see Figure 1; Hartigan et al. 1994; Ghez et al. 1997b; Brandner \& Zinnecker 1997; Prato 1998; White 1999; White \& Ghez 2000; Woitas et al. 2000 ). Of the 79 PMS secondaries with mass estimates, only one, GG Tau Bb, is a spectroscopically identified brown dwarf (White et al. 1999); this object is now the lowest mass, spectroscopically confirmed object in Taurus. In addition, 5 other objects are candidate brown dwarfs, requiring spectroscopic follow-up (DI Tau, HK Tau B, HBC 604 B, CZ Tau, Haro 6-28B; Meyer et al. 1997; Woitas et al. 2000). In summary, it is not possible to explain the factor of two more young companions with pre-main-sequence brown dwarfs.

The difference in companion star fraction between low stellar density star forming regions and the solar neighborhood provided the impetus for a number of both theoretical and observations studies. The theoretical studies model the effects of different physical properties, such as age, stellar density, initial cloud temperature, on the resulting companion star frequencies (e.g., Kroupa 1995; Kroupa et al. 1999; Durisen \& Sterzik 1994), while the observational studies survey regions with different properties to measure the resulting variation in companions with site. Observationally, the challenge is to compare similar types of samples, controlling for properties such as the primary star mass, limiting secondary star mass, and separation range (see, e.g., Duchene 1999). The results are varied. Results in the Hyades suggest a trend with age (Patience et al. 1998), whereas surveys of $\alpha$ Per, Praesepe, Pleiades, Orion show no evolution with time and a potential dependence on stellar density (e.g., Bouvier et al. 1997; Patience 2000; Duchene 2000; Prosser et al. 1994; Padgett et al. 1997; Petr et al. 1998; Simon et al. 1999). All these comparisons are made over a limited separation range. Some of the disagreements between these studies are explained when a larger range of separations is considered (Patience et al. 2000). As Figure 2 shows, PMS stars in loose associations, PMS stars in Orion, members of young open clusters, and solar-type stars in the solar neighborhood have significantly different distribution of separations. With these distributions, it is not surprising that comparisons of companion star fractions over different limited separation ranges give rise to different results.

Insight into the origin of the solar neighborhood stars can be obtained from the companion star frequency distributions, if the field G-dwarf distribution is assumed to be a superposition of distributions from the populations that contributed to it (e.g., Brandner \& Köhler 1998). In the analysis presented by 


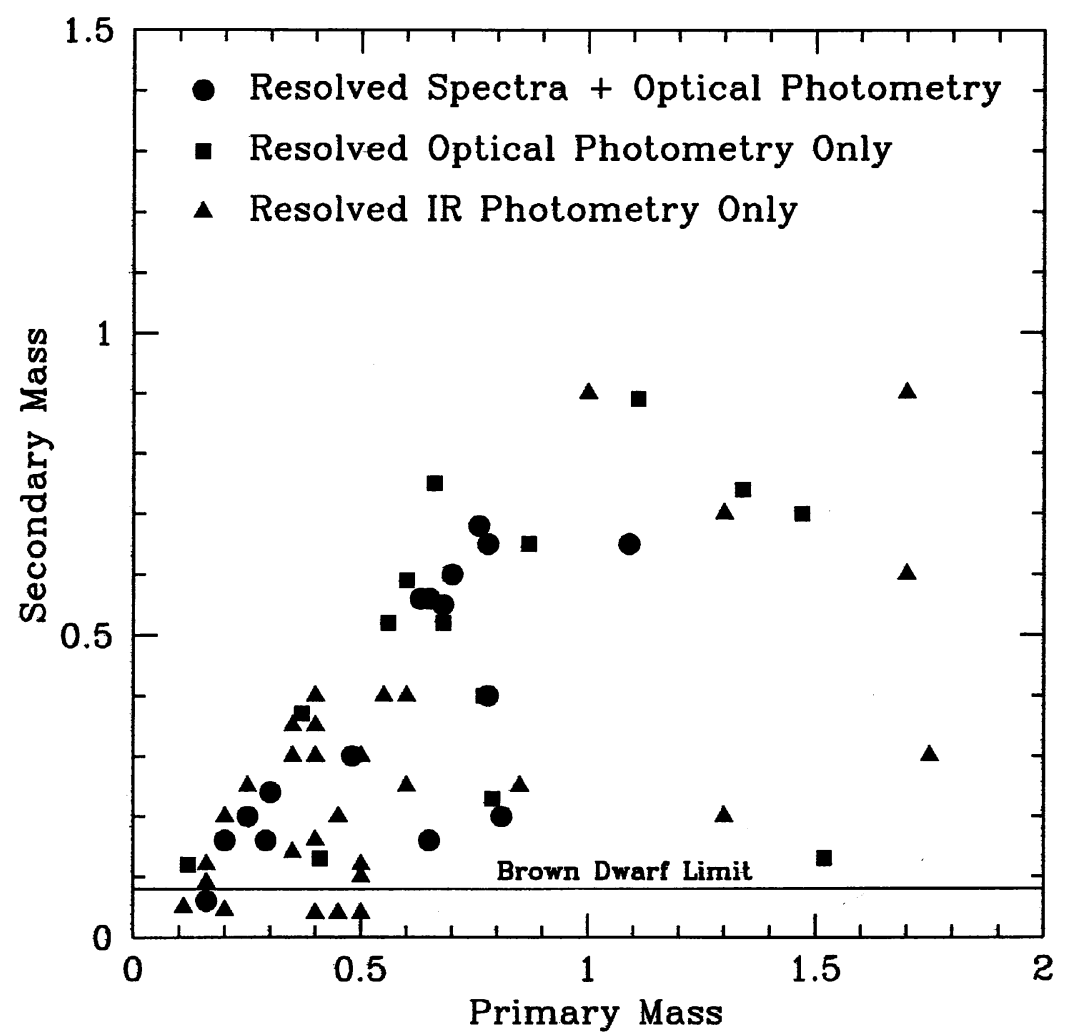

Figure 1. Secondary star mass vs. primary star mass for systems observed at multiple wavelengths (Hartigan 1994; Ghez et al. 1997; Brandner \& Zinnecker 1997; Prato 1998; White 1999; Woitas \& Leinert 2000). The majority of secondary stars in known T Tauri binaries have masses above the brown dwarf limit in spite of the relative ease of detecting such low mass companions at this early stage of evolution.

Patience et al. (2000), the G-dwarf distribution is scaled by a factor of 0.84 to account for the different mass ratio $\left(\mathrm{q}=M_{2} / M_{1}\right)$ sensitivities; where the $\mathrm{T}$ associations and young open clusters reach $\mathrm{q}=0.25$ and the corrected distribution of field G-dwarfs encompass $\mathrm{q}=0.1$. A fit of the combined $\mathrm{T}$ association and cluster distributions to the scaled field G-dwarfs suggests that $30 \pm 15 \%$ of the field binaries formed in dark clouds like the nearest T Tauri stars and the remaining $70 \pm 15 \%$ formed in the dense progenitors open clusters (Patience et al. 2000). This analysis suggests that a much lower fraction of the field is formed in a clustered environment than was previously suggested by star count studies, which obtained values as high as $96 \%$ (e.g., Lada et al. 1991). Consistent with the open cluster result, another recent study using wide proper motion systems in Orion as a probe of the populations that contributed to the field also sug- 
gests a lower percentage, $\sim 80 \%$, of systems formed in the clustered environment (Scally et al. 1999).

Analysis of the relationship between binary star formation in clustered vs. loose associations would be greatly assisted by surveys that reach separations of tens of $\mathrm{AU}$ in giant molecular clouds. Orion, the nearest giant molecular cloud, is $\sim 3$ times the distance to the nearest dark cloud complexes and therefore no published measurements have probed the separation range that shows an excess of companion stars. To reach this separation range, higher angular resolution is necessary; diffraction-limited imaging with the 10-m class telescopes affords the opportunity to probe binary star separations as small as $9 \mathrm{AU}$ at the distance of Orion. Such surveys, with angular resolution of $0 . " 05$ are just beginning (see Figure 3).

\section{Constraints on Star Formation Theories}

Binary star formation mechanisms fall into two broad categories, capture and fragmentation (see, e.g., review of Clarke 1996 or Clarke 2000). Several, although not all, of these theories have testable predictions for the observable properties of young binary stars. Of particular importance is how the companion star fraction and mass ratio $\left(\mathrm{q}=M_{2} / M_{1}\right)$ distributions depend upon stellar mass and binary star separation. Observations that probe these properties have just begun to make some progress. Below, I review some of the predictions and observations supporting and contradicting them (sometimes simultaneously!).

Capture is one of the oldest mechanisms proposed for binary star formation. Capture scenarios within a large cluster are generally ruled out as a dominant formation mechanism due to the rarity of capture encounters (Boss 1988). Moreover, the relative ages of binary star components formed in this manner are not necessarily coeval, which is inconsistent with the observation that the pre-main sequence binary star pairs are more co-eval than randomly paired members of Taurus (White 1999; White \& Ghez 2000). Capture or dynamical decay within small $\mathrm{N}$ clusters $(\mathrm{N}<\sim 10)$ is a much more efficient process in which binary pairs become gravitationally bound soon after the cluster formation (e.g., McDonald \& Clarke 1993, 1995; Sterzik \& Durisen 1998). In contrast to large cluster capture scenarios, this rapid capture mechanism yields binary pairs that are coeval. Another outcome of the small-N capture scenarios is that the companion star fraction is expected to increase with the primary star's mass. On the observational end, different trends are seen in different studies. Supporting evidence is found by Bouvier (2000), who reports an increase in companion star fraction for wide binaries with mass; the Herbig Ae/Be stars appear to have at least twice as many companions as the field G-dwarfs. Whereas, a new survey of M-dwarf $\mathrm{T}$ Tauri stars in Taurus finds no dependence with mass, with a companion star fraction between 3 and $250 \mathrm{AU}$ of $0.37 \pm 0.09$ compared to $0.46 \pm 0.04$ for the solar-like T Tauri stars (Ghez et al. 2000; see Figure 4). In more extreme contrast, the companion star fraction between 26 - $581 \mathrm{AU}$ appears to decrease with mass in a study of young open clusters (Patience 1999). It is therefore difficult to conclude what the true mass dependence for the companion star fraction is. More work needs to be done to establish what other factors may be responsible for the apparent contradictory results. 
Fortunately, small-N capture also has predications for the mass ratio distributions. In the first model of this class, small-N capture without disks (McDonald \& Clarke 1993), predicts that the mass ratio should rise towards unity. The mass ratio results are fortunately less contradictory between studies. None of the studies with measured mass ratios have mass ratios rising towards unity. For the young open clusters, the mass ratio distribution appears to be declining towards more equal mass systems (Patience 2000). The results for the younger stars in Taurus are somewhat flatter (Woitas \& Leinert 2000, White 1999), however the $2.2 \mu \mathrm{m}$ flux ratios suggest that there may be more equal mass systems in Taurus than in other regions (Ghez et al. 1997a; Duchene 1999). Adding disks to this model randomizes the companions and creates more small-q binaries, consistent with the observed distributions. However, the encounters that create the binary star pairs also preclude the formation of a circumbinary disk (Hall, Clarke \& Pringle 1996). Several circumbinary disk systems have already been directly or indirectly identified (e.g., Dutrey et al. 1994; Roddier et al. 1996; McCabe\& Ghez 2000; Jensen \& Mathieu 1997). Dynamical decay of few-body systems predicts a mass dependence for the mass-ratio distribution, with a high fraction of small-q systems occurring in systems with massive primary stars. This is supported by the young open clusters and has not been cleanly tested for the younger populations of stars. This leaves dynamical decay as the most attractive candidate from this class of models.

The fragmentation of either cloud cores during gravitational collapse (e.g., Bodenheimer 2000) or of a circumstellar disk (e.g., Bonnell 2000) constitutes the second class of mechanisms for forming binary stars. In general, fragmentation has the advantage that it naturally produces circumbinary disks (Bate \& Bonnell 1997; Bate 2000a). Disk instabilities that can lead to fragmentation have not yet lent themselves to any more clear testable predictions. However, only close binary stars can form in this scenario. Therefore one might expect to see a change in binary star properties at the scale of $\sim 100 \mathrm{AU}$, the typical size of a circumstellar disk. Without clear predictions, comparisons are not attempted here. For the specific case of scale-free core fragmentation, Clarke (1997) derived the following observable prediction: a mass ratio distribution that is independent or only weakly dependent upon the primary star mass. As described above, the observational results vary, but in many of the studies mass dependencies are observed. Numerical models of core fragmentation cannot currently simulate the full evolution from molecular cloud core to observable binary properties, however recent simulations of accretion onto protobinary fragments have generated several testable predictions (Bate 2000a). In an isolated star formation environment, closer and more massive binary stars are expected to have a higher fraction of more equal mass components. In Taurus, samples split at $\sim 160-180$ AU appear to show the predicted trend (Köhler \& Leinert 1998; White 1999). In contrast the young open cluster surveys show no such trends (Patience 1999). This, however, may be explained by the difference in competitive accretion in the isolated vs. clustered star formation environment (Bate 2000b). Further support for the isolated competitive accretion models is derived from the predicted circumstellar disk properties: if only one circumstellar disk exists, it is associated with the more massive component (a circumprimary disk). A corollary of this prediction is that the presence of a circumsecondary disk implies the existence of a circumprimary disk. This is strongly supported by observations 
in Taurus and Ophiuchus (Prato \& Simon 1997; Monin et al. 1998; White \& Ghez 1998; Duchene et al. 1999; White 2000; Prato \& Monin 2000). Therefore fragmentation appears to be a tenable mechanism and is in fact today the favored mechanism. It is important to note that capture and fragmentation may not be separate processes, as fragmentation may produce the small clusters that are the initial conditions of the capture scenarios (e.g., Boss 1998). However, there remains much work to be done on both the observational and theoretical end before a complete picture of binary star formation can be developed.

Acknowledgments. AG's research is supported by the Origins of Solar Systems Program and the Center for Adaptive Optics in Astronomy and Vision Science

\section{References}

Bate, M. 2000, MNRAS, 314, 33

Bate, M. 2001, this volume

Bate, M., \& Bonnell, I. A. 1997, MNRAS, 285, 33

Bodenheimer, P. 2001, this volume

Bonnell, I. A. 2001, this volume

Boss, A. P. 1988, ComAp, 12, 169

Bouvier, J. 2001, this volume

Bouvier, J., Rigaut, F., Nadeau, D. 1997, A\&A, 323, 139

Brandner, W., \& Köhler, R. 1998, ApJ, 499, 79

Brandner, W., \& Zinnecker, H. 1997, A\&A, 321, 220

Burrows, A., Hubbard, W. B., Lunine, J. L., Marley, M., \& Saumon, D. 1998, Protostars and Planets IV, ed. V. Mannings, A. Boss \& S. Russel

Clarke, C. 1996, in Evolutionary Processes in Binary Stars, ed. R. Wijers, M. Davies \& C. Tout (NATO ASI Series)

Clarke, C. 2001, this volume

Duchene, G. 1999, A\&A, 341, 547

Duchene, G. 2000, Ph.D., University of Grenoble

Durisen, R. H., \& Sterzik, M. F. 1994, A\&A, 286, 84

Dutrey, A., Guilloteau, S., \& Simon, M. 1994, A\&A, 286, 149

Ghez, A. M., Sand, D., White, R. J. 2000, in prep

Ghez, A. M., McCarthy, D. W., Patience, J. L., \& Beck, T. L. 1997a, ApJ, 481, 378

Ghez, A. M., Neugebauer, G., \& Matthews, K. 1993, AJ, 106, 2005

Ghez, A. M., White, R. J., \& Simon, M. 1997b, ApJ, 490, 353

Hall, S. M., Clarke, C. J., \& Pringle, J. E. 1997, MNRAS, 278, 303

Hartigan, P., Strom, K. M., \& Strom, S. E. 1994, ApJ, 427, 96

Köhler, R., \& Leinert, C. 1998, A\&A, 331, 977

Kroupa, P. 1995, MNRAS, 277, 1491

Kroupa, P., Petr, M. G., \& McCaughrean, M. J. 1999, New Astronomy, 4, 495 
Lada, E. A., Evans, N. J., Depoy, D. L., \& Gatley, I 1991, ApJ, 371, 171

Leinert, Ch., Zinnecker, H., Weitzel, N., Christou, J., Ridgway, S.T., Jameson, R., Haas, M., \& Lenzen, R. 1993, A\&A, 278, 129

McCabe, C., \& Ghez, A. M. 2001, this volume

McDonald, J. M., \& Clarke, C., J. 1993, MNRAS, 262, 800

McDonald, J. M., \& Clarke, C., J. 1995, MNRAS, 275, 671

Meyer, M. R., Beckwith, S. V. W., Herbst, T. M., \& Robberto, M. 1997, ApJ, 489, L173

Monin, J.-L., Menard, F., \& Duchene, G. 1998, A\&A, 339, 113

Padgett, D. L., Strom, S. E., \& Ghez, A. M. 1997, ApJ, 477, 705

Patience, J. L. 1999, Ph.D. UCLA

Patience, J. L., Ghez, A. M., Reid, I. N. 2000, in prep

Patience, J. L., Macintosh, B., Ghez, A. M. 2000, in prep

Prato, L. 1998, Ph.D., SUNY Stony Brook

Prato, L., \& Monin, J.-L. 2001, this volume

Prato, L., \& Simon, M. 1997, ApJ, 474, 455

Prosser, C. E., Stauffer, J. R., Hartmann, L., Soderblom, D. R., Jones, B. F., Werner, M. W., McCaughrean, M. J. 1994, ApJ, 421, 517

Roddier, C., Roddier, F., Northcott, M. J., Graves, J. E., \& Jim, K. 1996, ApJ, 463,326

Scally, A., Clarke, C., McCaughrean, M. J. 1999, MNRAS, 306, 253

Simon, M., Close, L. M., \& Beck. T. L. 1999, AJ, 117, 1375

Simon, M., Ghez, A. M., Leinert, Ch., Cassar, L., Chen, W. P., Howell, R. R., Jameson, R. F., Matthews, K., Neugebauer, G., \& Richichi, A. 1995, ApJ, 443, 625

Sterzik, M., \& Durisen, R. H. 1998, A\&A, 339, 95

White, R. J. 1999, Ph.D. UCLA

White, R. J., \& Ghez, A. M. 2000, in prep

White, R. J., \& Ghez, A. M. 1998, BAAS, 193, 7311

White, R. J., Ghez, A. M., Reid, I. N., \& Schultz, G. 1999, ApJ, 520, 811

Woitas, J., \& Leinert, Ch. 2000, A\&A, submitted 

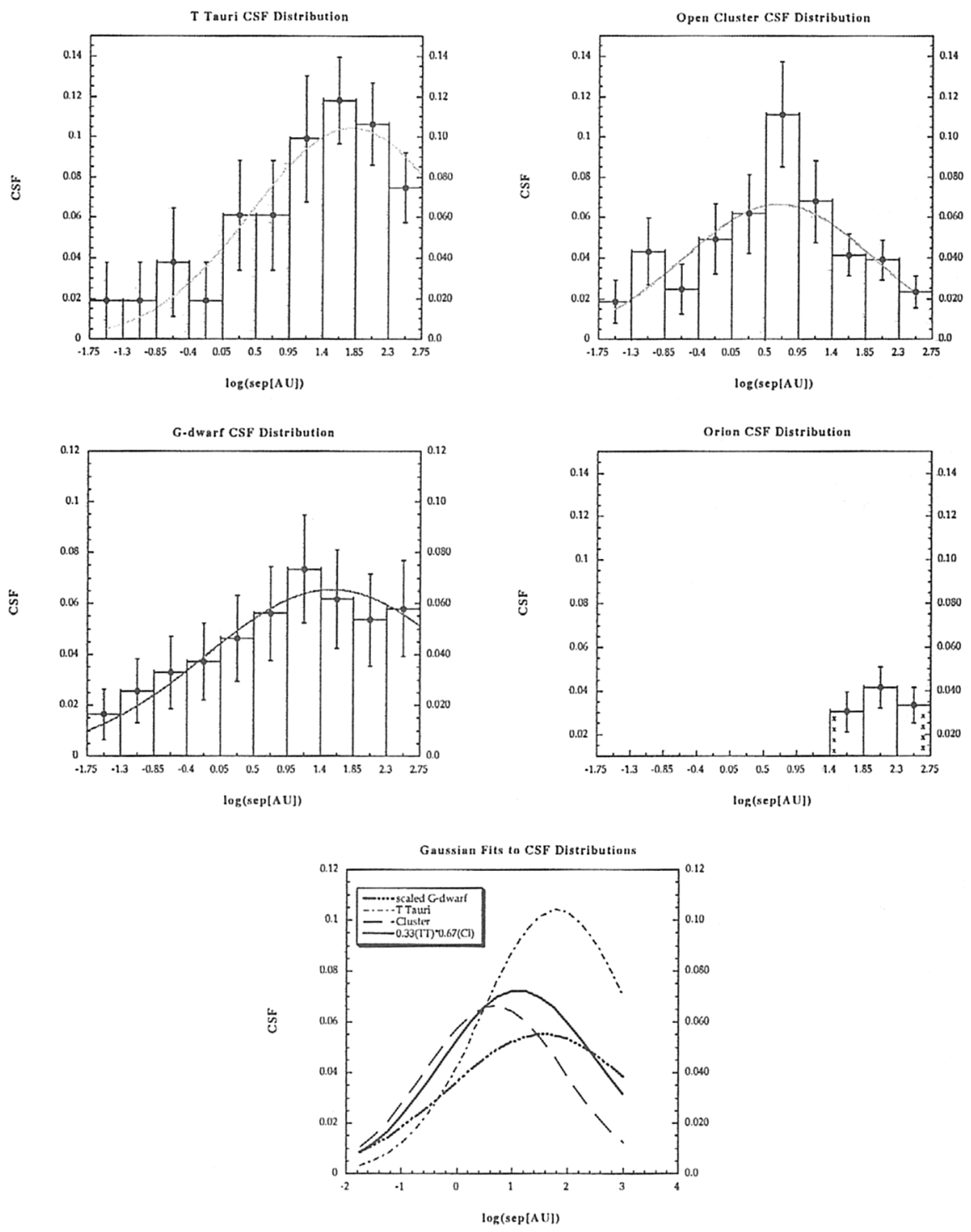

Figure 2. Companion star fraction (CSF) distributions spanning 0.02 - $581 \mathrm{AU}$ are constructed for (a; top left) PMS stars in loose associations ( $q>0.25)$, (b; top right) members of young open clusters $(q>0.25)$, (c; middle left) nearby solar-type stars ( $q>0.1)$, and (d; middle right) PMS stars in Orion. Significant differences in the populations' mean binary star separations exist. On the bottom row, the Gaussian fits to the first three distributions, with the G-dwarf distribution scaled by a factor of 0.84 to account for difference in mass ratio distributions. Combining the top two distribution suggests that $1 / 3$ of the field stars formed in loose associations and $2 / 3$ formed in the dense progenitors of open clusters. This figure is taken from Patience et al. (2000). 

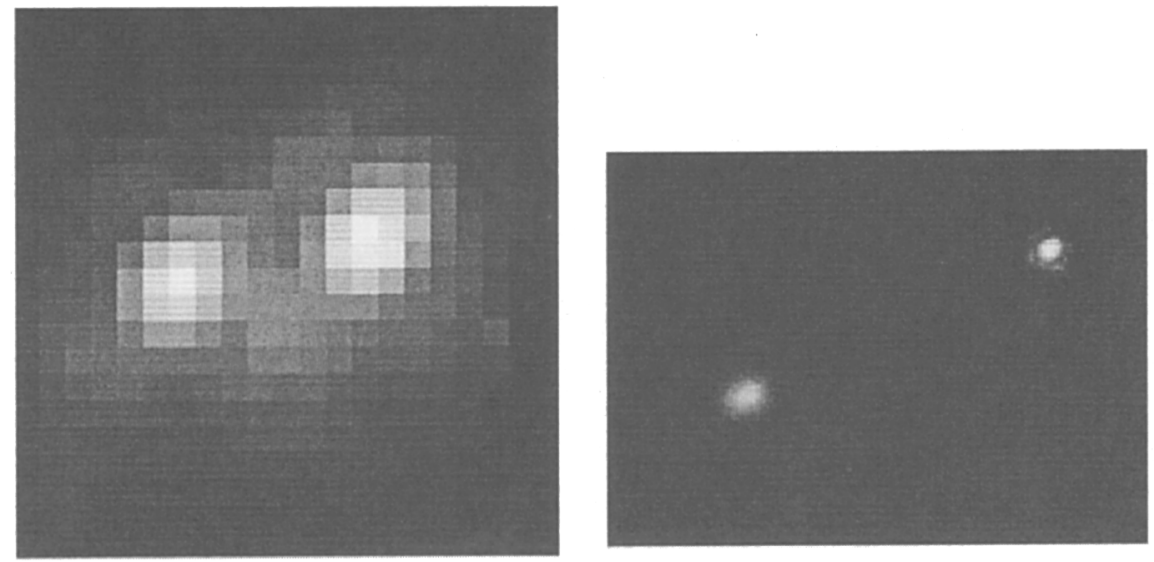

Figure 3. High angular resolution images in the Trapezium taken with Keck, which can probe scales as small as 9 AU (Patience, Macintosh, Ghez 2000). (a; left) A 0.15 binary stars (field of view 0".37 x 0 ". 37$)$ and (b; right) a newly detected extended object with a nearby point source (field of view $2 " .19 \times 1 " .58$ )
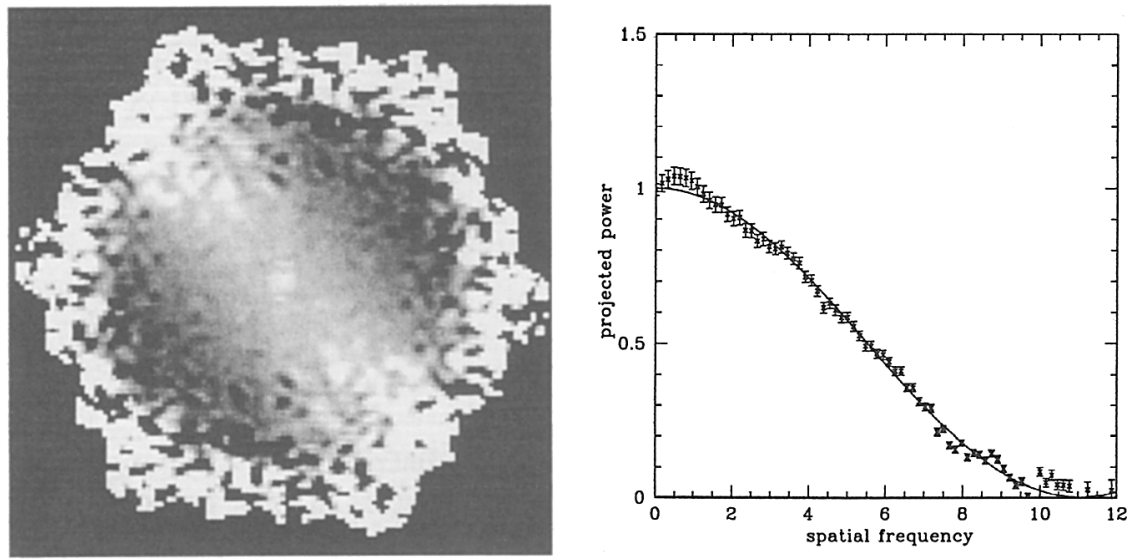

Figure 4. Power spectrum of one of the newly discovered M-dwarf $\mathrm{T}$ Tauri star in Taurus in (a; left) two-dimensions and ( $b$; right) projected into one-dimension. The binary star separation is $0 . " 02=3 \mathrm{AU}$ and the position angle has changed by 14 degrees in 1 year (Ghez et al. 2000). 